\title{
Epidemiology of bloodstream infections in patients with acute myeloid leukemia undergoing levofloxacin prophylaxis
}

\author{
Francesco Giuseppe De Rosa ${ }^{1 *}$, Ilaria Motta ${ }^{1}$, Ernesta Audisio ${ }^{2}$, Chiara Frairia $^{2}$, Alessandro Busca²,
} Giovanni Di Perri ${ }^{1}$ and Filippo Marmont ${ }^{2}$

\begin{abstract}
Background: Infections are a common cause of morbidity and mortality in patients with acute myeloid leukemia (AML). The evidence for efficacy of antibiotic prophylaxis in reducing the mortality rates and the incidence of bacterial infections was also reported by a systematic review published by Cochrane in 2012. The objective of our study was to report the incidence and the etiology of bloodstream infections in patients with AML undergoing levofloxacin prophylaxis during neutropenic episodes.

Methods: This was a retrospective study of patients with diagnosis of AML during 2001-2007.

Results: A total of 81 patients were included in the study. Two hundred and ninetyone neutropenic episodes were studied, of which 181 were febrile. Bacteria isolated from blood cultures were mostly Gram-positives during the induction (80\%) and Gram-negatives during the consolidation (72.4\%) phases of chemotherapy. Resistance to ciprofloxacin was found in $78.9 \%$ of isolated $E$. coli and it was higher during consolidation and higher than the hospital rate. The production of extended spectrum betalactamases (ESBL) in E. coli strains was reported in 12.1\%, below the reported hospital rate during the study period.

Conclusions: Regular microbiology surveillance is needed to better understand the impact of levofloxacin prophylaxis in neutropenic patients. Our study shows that Gram-positive bacteria are predominant during the induction phase of chemotherapy and Gram-negatives during the consolidation. The rate of fluoroquinolone resistance in the latter setting, even higher than the hospital rate, may suggest to reconsider levofloxacin prophylaxis.
\end{abstract}

Keywords: Acute myeloid leukemia, Neutropenia, Levofloxacin prophylaxis, Febrile neutropenia, Chemotherapy, Infections

\section{Background}

Acute myeloid leukemia (AML) is the most common acute leukemia in adults. Standard treatment is conventionally divided into the induction phase with anthracycline and cytarabine and consolidation therapy consisting of cycles of chemotherapy or stem cell transplantation $[1,2]$.

The survival rate is influenced by the prevention and management of infectious complications. Infections are a common cause of morbidity and mortality in patients with AML. The risk-assessment for infections in neutropenic

\footnotetext{
* Correspondence: francescogiuseppe.derosa@unito.it

${ }^{1}$ Department of Medical Sciences, Infectious Diseases, Amedeo di Savoia

Hospital, University of Turin, Corso Svizzera 164, 10149 Turin, Italy

Full list of author information is available at the end of the article
}

patients, according to the IDSA guidelines is classically divided into high risk (prolonged neutropenia, >7 days; neutrophils count $\leq 100 / \mathrm{mm}^{3}$; substantial concurrent comorbidity; clinically unstable) and low risk (neutropenia expected to resolve within 7 days; no active medical comorbidity, clinical stability at onset of the febrile episode; most of them are patients with solid tumors receiving conventional therapy) $[3,4]$.

Levofloxacin prophylaxis during neutropenia for high risk patients has been shown to be effective to prevent all infection related events by a study published in 2005 by GIMEMA group and confirmed by a Cochrane systematic review in $2012[5,6]$. However, empirical antifungal therapy and investigation for invasive fungal infections should

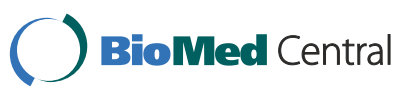


be considered for patients with persistent or recurrent fever after 4-7 days of antibiotics and whose duration of neutropenia is expected to be $>7$ days [7]. Preemptive antifungal management is acceptable as an alternative to empirical antifungal therapy in a subset of high-risk neutropenic patients $[8,9]$.

The primary objective of this study was to report the incidence of fever and clinically or microbiologically proven bacterial or fungal infections during neutropenic episodes of patients with AML undergoing levofloxacin prophylaxis. Our focus was on bloodstream infections [10].

\section{Methods}

This retrospective analysis was conducted in the Department of Hematology 2, at San Giovanni Battista Hospital in Turin between June 2001 and December 2007. The Ethics Committee approval was unnecessary due to the retrospective nature of the study and was waived with the approval of the Hospital Medical Direction for patients given prophylaxis with levofloxacin before 2006 . Beginning in 2006, consecutive adult patients with AML were prospectively included in the multicenter AML 02/ 06 (EudraCT number 2006-003817-429) study by our center, belonging to the Northern Italy Leukaemia Group (NILG) and approved by the Ethical Committee. All included patients were treated with standard induction chemotherapy ICE followed by post-remissional therapy with repeated consolidation courses with high dose cytarabine (HDAraC) plus peripheral progenitor cell support [11]. Each patient signed an informed consent to be included in the study and to receive chemotherapy, antinfective and nutritional therapy. As prescribed in the protocol, all patients received antibiotic prophylaxis with oral levofloxacin at $500 \mathrm{mg} /$ die, given during the induction phase from the day of hospitalization until the recovery of blood neutrophils count over $1000 / \mu \mathrm{l}$, after chemotherapy. In the following courses of chemotherapy levofloxacin at the same dosage was administered from first day after the end of chemotherapy until the recovery of blood neutrophils count over 1000/ $\mu$ l. Antifungal prophylaxis was administered to all patients with oral itraconazole $200 \mathrm{mg}$ twice a day.

In patients with neutropenia $\left(\leq 500 / \mathrm{mm}^{3}\right)$ and fever (defined as external temperature $\geq 38^{\circ} \mathrm{C}$ ) the following baseline diagnostic investigations were performed: chest $\mathrm{X}$-rays, blood cultures, sputum and urine cultures, serum galactomannan antigen, Streptococcus pneumoniae and Legionella spp. urinary antigens. Empiric treatment, based on international recommendation at the time, could vary among the centres, according to local epidemiology. Neutropenic febrile patients were treated with empirical antibiotic therapy with piperacillin-tazobactam or meropenem. Vancomycin and/or amikacin were added if fever was deemed to be complicated, such as suggestion of intravascular catheter-related infection, MRSA colonization, hypotension and/or organ failure.

Data collected included demographics, presence and characteristics of central venous catheter (type, site, insertion and removal), duration of antibiotic prophylaxis received, description of febrile episodes (duration, initial neutrophil count, blood pressure, $\mathrm{SO} 2 \%$, respiratory rate, body temperature), type and length of antibiotics, results of investigations (chest X-ray and CT scan, ultrasounds, brain CT scan) and microbiology tests (site of infection, analyzed material, isolation of microorganism).

Data was entered into an electronic database and analyzed with Microsoft Excel. Statistical analysis was performed with STATA 11 program (Stata Corporation, USA). Chi-square test was used for categorical variables. Continuous variables were compared by Student's t-test if normally distributed and the Mann-Whitney U-test if non-normally distributed. All $p$ values were two sided, $p$ value of $<0.05$ was considered significant. Values for continuous and categorical variables are expressed as the mean \pm SD and median (IQR) or percentage of the group from which they are derived, respectively.

\section{Results}

A total of 81 patients with diagnosis of AML were observed during the study period (46 males and 35 females). The median age was $49.7 \pm 11.4$ years (range 23-69 years). There were 291 neutropenic episodes, 81 $(27,8 \%)$ during in the induction and $210(72,2 \%)$ during the consolidation phase; fever was recorded in 181 episodes, 69 during induction (85.2\%) and 112 (53.4\%) during consolidation cycles. Of the latter 112 episodes $32(28,6 \%)$ were during the second cycle; 25 (22,3\%), 27 (24,1\%), 17 $(15,2 \%)$ and $11(9,8 \%)$ during the third, fourth, fifth and sixth consolidation cycle, respectively. The characteristics are reported in Table 1 . The mean duration of neutropenia was 14 (range 13-19) days for the induction phase (ICE), 7 (range 5-11) and 5 (range 4-7) days for the second and

Table 1 Comparison of neutropenic episodes according to clinical or microbiological confirmation of infection

\begin{tabular}{lccc}
\hline & $\begin{array}{c}\text { Induction } \\
\mathbf{( N = 8 1 )}\end{array}$ & $\begin{array}{c}\text { Consolidation } \\
(\mathbf{N}=\mathbf{2 1 0})\end{array}$ & $\boldsymbol{p}$ value \\
\hline Fever & $27(33,4 \%)$ & $40(19,1 \%)$ & $<0.001$ \\
Clinical diagnosis & $9(11,1 \%)$ & $5(2,4 \%)$ & 0.002 \\
Microbiological diagnosis & $24(29,6 \%)$ & $66(31,4 \%)$ & 0.766 \\
Possible IFI & $2(2,5 \%)$ & & \\
Probable IFI & $3(3, \%)$ & & \\
Definite IFI & $4(4,9 \%)$ & $1(0,5 \%)$ & $<0.001$ \\
Total & $\mathbf{6 9}$ & $\mathbf{1 1 2}$ & $<0.001$ \\
Patients without fever & $12(14,8 \%)$ & $98(46.6 \%)$ & \\
\hline
\end{tabular}


the third consolidation cycle, respectively. The median duration of neutropenia following the peripheral stem cells transplantation was 12 days. The median number of days of fever was significantly higher in the induction phase than in the consolidation chemotherapy ( 9 vs 4 days, $p<0.001$ ).

All the patients carried a Hohn central venous catheter both in induction and in consolidation phase. Twentyfour patients underwent allogenic transplantation (29,6\%). In twenty-six patients $(32,1 \%)$ high-dose of citarabine was followed by peripheral stem cells transplantation (preceded by rescue of peripheral blood progenitor cells).

Pneumonia (fungi included) was the most common clinical manifestation: during the induction chemotherapy in 69 patients with fever (19 cases, 27,5\%) and during the consolidation chemotherapy in 112 febrile neutropenia episodes (12 cases, 10,7\%).

Piperacillin/tazobactam was mainly administered as empirical treatment, followed by meropenem or ceftazidime in association with amikacin; vancomycin was empirically added in 21, 11 and 4 cases, respectively. Resolution of fever during induction chemotherapy was observed in $47,6 \%$ of patients treated with piperacillin/ tazobactam, in $42,9 \%$ with ceftazidime and amikacin and $40 \%$ with meropenem. A higher rate of clinical success (disappearance of fever or resolution of clinical manifestation) was reported during the consolidation phase in $91,7 \%$ of patients treated with meropenem, $88,9 \%$ treated with meropenem and glicopeptide, $75 \%$ treated with ceftriaxone and in $68,7 \%$ of those who received ceftazidime and amikacin. The lower duration of neutropenia during the consolidation probably explained the higher rate of fever disappearance in the consolidation phase compared with the induction phase $(79.5 \%$ versus $52.2 \%, p=0.00018$ ).

Amongst the febrile neutropenic episodes, 29\% were associated with bacteraemia during the induction (20 positive blood cultures for bacteria over 69 febrile neutropenic episodes) and 51\% during the consolidation phase (59 positive blood cultures for bacteria over 112 febrile neutropenic episodes).

Amongst bloodstream isolates, all coagulase-negative Staphylococci were methicillin-resistant and resistant to fluoroquinolones. Enterococcus faecalis isolates were resistant to ampicillin but sensitive to vancomycin and teicoplanin. Amongst Enterococcus faecium, vancomycin and teicoplanin were active in vitro against two out of three isolates $(66,6 \%)$. The resistance to fluoroquinolones was also complete amongst the strains of Corynebacterium spp and it was $78 \%$ in Escherichia coli, which were ESBL producers in $12.1 \%$ of cases $(\mathrm{N}=4)$, all in the consolidation phases.

The main finding of this study was that bloodstream isolates were predominantly represented by Gram- positives, $80 \%$ vs $27,6 \%$ ( 16 cases out of 20 vs 17 cases out of 59) or by Gram-negatives, $20 \%$ vs $72,4 \%$ (4 cases out of 20 vs 42 cases out of 59) during the induction or consolidation phases, respectively $(p<0.001)$ (Table 2).

There were ten cases of fungal infections, all but one during the induction phase ( 2 possible, 3 probable and 5 proven). Proven invasive pulmonary aspergillosis were diagnosed in four cases.

During the study six patients died $(7,4 \%)$ : four with invasive fungal disease, one with Legionella pneumonia and refractory disease and one with refractory disease without signs of infection.

\section{Discussion}

Levofloxacin prophylaxis of febrile episodes in neutropenic patients has been shown to be effective by the GIMEMA study in 2005 [5]. In the same paper the authors also emphasized the need for surveillance for the possible development of antibiotic resistance. In the following years both IDSA guidelines [3] and a Cochrane review of 109 randomized trials [6] confirmed the utility of levofloxacin prophylaxis in selected patient populations with oncohaematologic disease. In this study we retrospectively evaluated neutropenic episodes in patients with AML who received levofloxacin prophylaxis. We found a statistically significant prevalence of Gram-positive bacteria during induction chemotherapy and Gram-negatives during consolidation phase.

Table 2 Etiology of positive blood cultures during the induction and consolidation phase

\begin{tabular}{|c|c|c|}
\hline & $\begin{array}{l}\text { Gram-positives } \\
(\mathrm{N}=33)\end{array}$ & $\begin{array}{l}\text { Gram-negatives } \\
(\mathrm{N}=46)\end{array}$ \\
\hline Induction ( $N=81)$ & S. epidermidis (11) & E. cloacae (2) \\
\hline \multirow{3}{*}{$\begin{array}{l}\text { Neutropenic fever } \\
\text { episodes }=69\end{array}$} & E. faecium (3) & E. coli (1) \\
\hline & S. mitis (1) & \multirow[t]{2}{*}{ K. pneumoniae (1) } \\
\hline & Corynebacterium spp. (1) & \\
\hline Total $(\mathrm{N}=20)$ & 16 & 4 \\
\hline Consolidation ( $N=210)$ & S. epidermidis (8) & E. coli $(32)$ \\
\hline \multirow{6}{*}{$\begin{array}{l}\text { Neutropenic fever } \\
\text { episodes }=112\end{array}$} & MRSA (3) & P. aeruginosa (3) \\
\hline & S. hominis (3) & E. cloacae (2) \\
\hline & Corynebacterium spp (2) & S. maltophilia (2) \\
\hline & \multirow[t]{3}{*}{ E. faecalis (1) } & S. marcescens (1) \\
\hline & & Citrobacter (1) \\
\hline & & K. pneumoniae (1) \\
\hline Total $(\mathrm{N}=59)$ & 17 & 42 \\
\hline
\end{tabular}


The use of prophylactic levofloxacin during every cycle of chemotherapy may have a role in selection of isolated strains, as suggested by Bucaneve et al. in their seminal paper. A recent systematic review of RCTs and quasiRCTs by Cochrane in 2012 demonstrated that levofloxacin prophylaxis significantly reduced all-cause mortality and infection-related mortality, compared to placebo and also significantly reduced febrile episodes [6]. According to those results, quinolone prophylaxis did not increase the incidence of Gram-positive bacteraemia and, more importantly, there was no significant differences in the number of patients developing infections caused by organisms resistant to quinolones.

The spectrum of coverage of fluoroquinolones may account for a lower incidence of bacteraemia by Gramnegatives in the induction phase observed in our study, where the isolation of Gram-positive bacteria may be explained by partial efficacy of the prophylaxis regimen, excluding multidrug resistant strains. The significantly higher prevalence of Gram-negatives during the consolidation phase may be explained by the previous administration of levofloxacin prophylaxis, since as many as $73,7 \%$ of Gram-negatives were resistant to fluoroquinolones (78.9\% of E. coli were resistant to ciprofloxacin), corresponding to $59,6 \%$ of the total number of Gram-positives and Gram-negatives. In the induction phase Gramnegatives bacteria resistant to fluoroquinolones accounted for only $15,4 \%$ of the total number of bacteria.

Of course we cannot exclude that differences in etiology could be due to other factors, such as comorbidities and hospital stay. However, during the study period there was a lower rate of fluoroquinolones resistance amongst bloodstream E. coli hospital isolates (29-38\%) and there were few cases of bloodstream infections caused by ESBL-producers $E$. coli, with resistance to fluoroquinolones in $76.3 \%$ of strains [12]. Amongst patients undergoing allogenic transplant and levofloxacin prophylaxis, coagulase-negative staphylococci were the most commonly isolated Gram-positive pathogens and E. coli was the most commonly isolated Gramnegative bacteria [13]. Resistance to fluoroquinolones was $87 \%$ amongst Gram-positive isolates and 50\% amongst Gram-negative rods. The issue of growing rate of fluoroquinolone resistance and ESBL production by Gram-negatives may require special attention even at hospital admission in selected patient population where a clinical risk score may be useful [14].

However, since fever disappeared more rapidly during the consolidation phases of chemotherapy, because of reduced duration of neutropenia together with the probable effect of empiric antibiotic treatment, the administration of levofloxacin prophylaxis might be discussed under the view of the local epidemiology, especially when multiple or prolonged administration is expected.

\section{Conclusions}

Fluoroquinolone prophylaxis is frequently used worldwide in high-risk neutropenic patients. However, few reports have studied the rate of resistance amongst Gram-positive and Gram-negative bloodstream isolates during induction and consolidation phases. In our study the rate and resistance of Gram-negatives was significantly higher during the consolidation phase, where the duration of neutropenia is lower and the resolution of fever after empiric antibiotic treatment is higher. If these data will be confirmed, levofloxacin prophylaxis might be at least less extensively administered during the consolidation chemotherapy. Constant monitoring for fluoroquinolone resistance amongst Gram-negative bacteria is required to preserve the efficacy of levofloxacin prophylaxis.

\section{Abbreviations}

AML: Acute myeloid leukemia; ESBL: Extended spectrum beta lactamases; IFI: Invasive fungal infection.

\section{Competing interests}

The authors declare that they have no competing interests.

\section{Authors' contributions}

FDR, FM, EA contributed to study design, data collection, interpretation of data and statistical analysis. CF contributed to data collection. FDR, IM, FM, $E A$ drafted the first version of the manuscript and finalized the manuscript. $A B, G D P$ contributed to study design, supervision and critical revision of the manuscript for intellectual content. All authors read and approved the final manuscript.

\section{Author details}

${ }^{1}$ Department of Medical Sciences, Infectious Diseases, Amedeo di Savoia Hospital, University of Turin, Corso Svizzera 164, 10149 Turin, Italy. ${ }^{2}$ SC Haematology II, AOU Città della Salute e della Scienza, San Giovanni Battista Molinette Hospital, Turin, Italy.

Received: 4 February 2013 Accepted: 18 November 2013 Published: 1 December 2013

\section{References}

1. Han LN, Zhou J, Shuringa JJ, Vellenga E: Treatment strategies in acute myeloid leukemia. Chin Med J (Engl) 2011, 124(9):1409-1421.

2. Roboz GJ: Novel approaches to the treatment of acute myeloid leukemia. Hematology Am Soc Hematol Educ Program 2011, 2011:43-50.

3. Freifeld AG, Bow EJ, Sepkowitz KA, et al: IDSA guidelines clinical practice guideline for the use of antimicrobial agents in neutropenic patients with cancer: 2010 update by the infectious diseases society of America. Clin Infect Dis 2010, 52(4):e56-e93.

4. Rolston KV: New trends in patient management: risk-based therapy for febrile patients with neutropenia. Clin Infect Dis 1999, 29:515-521.

5. Bucaneve G, Micozzi A, Menichetti F, et al: Levofloxacin to prevent bacterial infection in patients with cancer and neutropenia. N Engl J Med 2005, 353:977-987.

6. Gafter-Gvili A, Fraser A, Paul M, et al: Antibiotic prophylaxis for bacterial infections in afebrile neutropenic patients following chemotherapy. Cochrane Database Syst Rev 2012, 1:25-27.

7. Denning DW: Invasive aspergillosis. Clin Infect Dis 1998, 26:781-805.

8. Ascioglu S, Rex JH, de Pauw B, et al: Defining opportunistic invasive fungal infections in immunocompromised patients with cancer and hematopoietic stem cell transplants: an international consensus. Clin Infect Dis 2002, 34:7-14.

9. Corey L, Boeckh M: Persistent fever in patients with neutropenia. N Engl J Med 2002, 346:222-224. 
10. Chen CY, Tsay W, Tang GL, et al: Epidemiology of bloodstream infections in patients with hematological malignancies with and without neutropenia. Epidemiol Infect 2010, 138:1044-1051.

11. Intermesoli T, Oldani E, Rossi G, et al: Two-step response-oriented induction predicts long-term outcome of adult patients with standard- and high-risk acute myeloid leukaemia (AML): a Northern Italy Leukaemia Group (NILG) study. In Abstracts of the Fifteenth EHA Annual Meeting. Barcelona: Abstract 649. European Hematology Association; 2010.

12. De Rosa FG, Pagani N, Fossati $L$, et al: The effect of inappropriate therapy on bacteremia by ESBL-producing bacteria. Infection 2011, 39(6):555-561.

13. Busca A, Cavecchia I, Locatelli F, et al: Blood stream infections after allogeneic stem cell transplantation: a single-center experience with the use of levofloxacin prophylaxis. Transpl Infect Dis 2012, 14(1):40-48.

14. Tumbarello M, Trecarichi EM, Bassetti M, et al: Identifying patients harboring extended-spectrum-beta-lactamase-producing Enterobacteriaceae on hospital admission: derivation and validation of a scoring system. Antimicrob Agents Chemother 2011, 55(7):3485-3490.

doi:10.1186/1471-2334-13-563

Cite this article as: De Rosa et al: Epidemiology of bloodstream infections in patients with acute myeloid leukemia undergoing levofloxacin prophylaxis. BMC Infectious Diseases 2013 13:563.

\section{Submit your next manuscript to BioMed Central and take full advantage of:}

- Convenient online submission

- Thorough peer review

- No space constraints or color figure charges

- Immediate publication on acceptance

- Inclusion in PubMed, CAS, Scopus and Google Scholar

- Research which is freely available for redistribution 